\title{
A Note on Numerical Radius Operator Spaces
}

\author{
Yuanyi Wang1, Yafei Zhao² \\ ${ }^{1}$ College of Science and Technology, Ningbo University, Ningbo, China \\ ${ }^{2}$ Department of Mathematics, Zhejiang International Studies University, Hangzhou, China \\ Email: wngyny@163.com, zhaoyafei zju@163.com
}

How to cite this paper: Wang, Y.Y. and Zhao, Y.F. (2019) A Note on Numerical Radius Operator Spaces. Journal of Applied Mathematics and Physics, 7, 1251-1262. https://doi.org/10.4236/jamp.2019.76085

Received: May 14, 2019

Accepted: June 16, 2019

Published: June 19, 2019

Copyright (c) 2019 by author(s) and Scientific Research Publishing Inc. This work is licensed under the Creative Commons Attribution International License (CC BY 4.0).

http://creativecommons.org/licenses/by/4.0/

\begin{abstract}
In this paper, we first study some $\mathcal{W}$-completely bounded maps between various numerical radius operator spaces. We also study the dual space of a numerical radius operator space and show that it has a dual realization. At last, we define two special numerical radius operator spaces MinE and MaxE which can be seen as a quantization of norm space $E$.
\end{abstract}

\section{Keywords}

Numerical Radius Operator Space, Dual Space, Quantization

\section{Introduction and Preliminaries}

The theory of operator space is a recently arising area in modern analysis, which is a natural non-commutative quantization of Banach space theory. An operator space is a norm closed subspace of $\mathcal{B}(\mathcal{H})$. The study of operator space begins with Arverson's [1] discovery of an analogue of the Hahn-Banach theorem. Since the discovery of an abstract characterization of operator space by Ruan [2], there have been many more applications of operator space to other branches in functional analysis. Effros and Ruan studied the mapping spaces $C B(V, W)$ in [3] and the minimal and maximal operator spaces in [4]. The fundamental and systematic developments in the theory of tensor product of operator spaces can be found in [5] [6]. The tensor products provide a fruitful approach to mapping spaces and local property. For example, Effros, Ozawa and Ruan [7] showed that an operator space $V$ is nuclear if and only if $V$ is locally reflexive and $V^{* *}$ is injective. Dong and Ruan [8] showed that an operator space $V$ is exact if and only if $V$ is locally reflexive and $V^{* *}$ is weak ${ }^{*}$ exact. In [9], Han showed that an operator space $V$ satisfies condition $C$ if and only if it satisfies conditions $C^{\prime}$ and $C^{\prime \prime}$. Based on the work of Han, Wang [10] gave a characterization of condition $C_{\wedge}^{\prime}$ on the operator spaces. Amini, Medghalchi and Nikpey [11] proved that an 
injective operator space is global exactness if and only if it is reflexive. The readers may refer to [12] [13] for the basics on operator spaces.

Recently, some new algebraic structures derived from operator spaces also have been intensively studied. An operator system is a matrix ordered operator space which plays a profound role in mathematical physics. Kavruk, Paulsen, Todorov and Tomforde gave a systematic study of tensor products and local property of operator systems in [14] [15]. In [16], Luthra and Kumar showed that an operator system is exact if and only if it can be embedded into a Cuntz algebra. The numerical radius operator space is also an important algebraic structure which is introduced by Itoh and Nagisa [17] [18]. The conditions to be a numerical radius space are weaker than the Ruan's axiom for an operator space. It is shown that there is a $\mathcal{W}$-complete isometry from a numerical radius operator space into a Hilbert space with numerical radius norm. They also studied many relations between the operator spaces and the numerical radius operator spaces. The category of operator space can be regarded as a subcategory of numerical radius operator space.

We now recall some concepts needed in our paper. An (abstract) operator space is a complex linear space $V$ together with a sequence of norms $\mathcal{O}_{n}(\cdot)$ on the $n \times n$ matrix space $M_{n}(V)$ for each $n \in \mathbb{N}$, which satisfies the following Ruan's axioms OI, OII:

$$
\begin{gathered}
\text { OI }: \mathcal{O}_{m+n}\left(\left(\begin{array}{cc}
v & 0 \\
0 & w
\end{array}\right)\right)=\max \left\{\mathcal{O}_{m}(v), \mathcal{O}_{n}(w)\right\} ; \\
\text { OII }: \mathcal{O}_{n}(\alpha v \beta) \leq\|\alpha\| \mathcal{O}_{m}(v)\|\beta\|
\end{gathered}
$$

for all $v \in M_{m}(V), w \in M_{n}(V)$ and $\alpha \in M_{n, m}(\mathbb{C}), \beta \in M_{m, n}(\mathbb{C})$. If $V$ is an (abstract) operator space, then there is a complete isometry $\Psi$ from $V$ to $\mathcal{B}(\mathcal{H})$, that is, $\left\|\left[\Psi\left(v_{i, j}\right)\right]\right\|_{n}=\mathcal{O}_{n}\left(\left[v_{i, j}\right]\right)$ for all $\left[v_{i, j}\right] \in M_{n}(V), n \in \mathbb{N}$.

An abstract numerical radius operator space is a complex linear space $V$ together with a sequence of norms $\mathcal{W}_{n}(\cdot)$ on the $n \times n$ matrix space $M_{n}(V)$ for each $n \in \mathbb{N}$, which satisfies the following axioms WI, WII:

$$
\begin{gathered}
\mathrm{WI}: \mathcal{W}_{m+n}\left(\left(\begin{array}{ll}
v & 0 \\
0 & w
\end{array}\right)\right)=\max \left\{\mathcal{W}_{m}(v), \mathcal{W}_{n}(w)\right\} ; \\
\text { WII }: \mathcal{W}_{n}(\alpha v \alpha) \leq\|\alpha\|^{2} \mathcal{W}_{m}(v)
\end{gathered}
$$

for all $v \in M_{m}(V), w \in M_{n}(W)$ and $\alpha \in M_{n, m}(\mathbb{C})$. Let $\omega(\cdot)$ be the numerical radius norm on $\mathcal{B}(\mathcal{H})$. If $V$ is an abstract numerical radius operator space, then there is a $\mathcal{W}$-complete isometry $\Phi$ from $\left(V, \mathcal{W}_{n}\right)$ to $\left(\mathcal{B}(\mathcal{H}), \omega_{n}\right)$, that is, $\omega_{n}\left(\Phi\left(v_{i, j}\right)\right)=\mathcal{W}_{n}\left(\left[v_{i, j}\right]\right)$ for all $\left[v_{i, j}\right] \in M_{n}(V), n \in \mathbb{N}$. Given a numerical radius operator $\left(V, \mathcal{W}_{n}\right)$, we can define an operator space $\left(V, \mathcal{O}_{n}\right)$ by

$$
O W: \frac{1}{2} \mathcal{O}_{n}(v)=\mathcal{W}_{2 n}\left(\left(\begin{array}{ll}
0 & v \\
0 & 0
\end{array}\right)\right)
$$

for all $v \in M_{n}(V)$ 
Given abstract numerical radius operator spaces (or operator spaces) $V, W$ and a linear map $\varphi$ from $V$ to $W, \varphi_{n}$ from $M_{n}(V)$ to $M_{n}(W)$ is defined to be $\varphi_{n}\left(\left[v_{i, j}\right]\right)$ for each $\left[v_{i, j}\right] \in M_{n}(V), n \in \mathbb{N}$. We use a simple notation for the norm of $v=\left[v_{i, j}\right] \in M_{n}(V)$ to be $\mathcal{W}(v)$ (resp. $\left.\mathcal{O}(v)\right)$ instead of $\mathcal{W}_{n}(v)$ (resp. $\mathcal{O}_{n}(v)$ ), and for the norm of $f \in M_{n}(V)^{*}$ to be

$$
\mathcal{W}^{*}(f)=\sup \left\{|f(v)|: v=\left[v_{i, j}\right] \in M_{n}(V), \mathcal{W}(v) \leq 1\right\} .
$$

We denote the norm $\varphi_{n}$ by

$$
\begin{gathered}
\mathcal{W}\left(\varphi_{n}\right)=\sup \left\{\mathcal{W}\left(\varphi_{n}(v)\right): v=\left[v_{i, j}\right] \in M_{n}(V), \mathcal{W}(v) \leq 1\right\} \\
\text { (resp. } \left.\mathcal{O}\left(\varphi_{n}\right)=\sup \left\{\mathcal{O}\left(\varphi_{n}(v)\right): x=\left[v_{i, j}\right] \in M_{n}(V), \mathcal{O}(v) \leq 1\right\}\right) .
\end{gathered}
$$

The $\mathcal{W}$-completely bounded norm (resp. completely bounded norm) of $\varphi$ is defined to be $\mathcal{W}(\varphi)_{c b}=\sup \left\{\mathcal{W}\left(\varphi_{n}\right): n \in \mathbb{N}\right\},\left(\right.$ resp. $\left.\mathcal{O}(\varphi)_{c b}=\sup \left\{\mathcal{O}\left(\varphi_{n}\right): n \in \mathbb{N}\right\}\right)$. We say $\varphi$ is $\mathcal{W}$-completely bounded (resp. completely bounded) if $\mathcal{W}(\varphi)_{c b}<\infty$ (resp. $\left.\mathcal{O}(\varphi)_{c b}<\infty\right)$, and $\varphi$ is $\mathcal{W}$-completely contractive (resp. completely contractive) if $\mathcal{W}(\varphi)_{c b} \leq 1$ (resp. $\left.\mathcal{O}(\varphi)_{c b} \leq 1\right)$. We call $\varphi$ is a $\mathcal{W}$ -complete isometry (resp. complete isometry) if $\mathcal{W}\left(\varphi_{n}(v)\right)=\mathcal{W}(v)$ (resp. $\left.\mathcal{O}\left(\varphi_{n}(v)\right)=\mathcal{O}(v)\right)$ for each $x \in M_{n}(V), n \in \mathbb{N}$.

In Section 2, we study the bounded maps on finite dimension numerical radius operators and commutation $\mathrm{C}^{\star}$-algebras. We prove these maps are all $\mathcal{W}$ -completely bounded. In Section 3, we study the dual space of a numerical radius operator space and prove its dual space has a dual realization on a Hilbert space $\mathcal{H}$. In Section 4, we define the numerical radius operator spaces MinE and MaxE for a normed space $E$, and prove that $(\operatorname{Max} E)^{*}=\operatorname{MinE}^{*}$ and $\operatorname{MaxE}^{*}=(\operatorname{MinE})^{*}$.

In order to improve the readability of the paper, we give an index of notation:

\begin{tabular}{cc}
\hline & Index of Notation \\
\hline $\mathcal{B}(\mathcal{H})$ & Hilbert space \\
$M_{m, n}(V)$ & $m$ by $n$ matrix space over $V$ \\
$C_{0}(\Omega)$ & Space of continuous complex functions vanishing at $\infty \quad$ on $\Omega$ \\
$\|\alpha\|$ & Matrix norm of $\alpha$ \\
$\langle v, w\rangle$ & Scalar pairing of matrices \\
$\langle\langle v, w\rangle\rangle$ & Matrix pairing of matrices \\
$\left(V, \mathcal{O}_{n}\right)$ & Operator space \\
$\left(V, \mathcal{W} W_{n}\right)$ & Numerical radius operator space \\
$\omega(\cdot)$ & Numerical radius norm on $\mathcal{B}(\mathcal{H})$ \\
$B(V, W)$ & Space of bounded mappings \\
$B^{\sigma}(V, W)$ & Space of $w^{*}$-bounded mappings \\
$C B(V, W)$ & Space of completely bounded mappings \\
$\mathcal{W} C B(V, W)$ & Space of $\mathcal{W}$-completely bounded mappings
\end{tabular}




\section{Bound Linear Maps}

In this section, we study some bounded linear maps on the numerical radius operator spaces.

Proposition 2.1. If $\left(V, \mathcal{O}_{n}\right)$ is an operator space and $\left(V, \mathcal{W}_{n}\right)$ is a numerical radius operator space satisfies $\|v\|=1$, then the mapping

$$
\theta_{v}: C \rightarrow V: \alpha \rightarrow \alpha v
$$

is $\mathcal{W}$-completely isometric.

Proof. Since $\mathcal{W}_{\max }(\mathbb{C})=\omega(\mathbb{C})$, by Lemma 3.8 and 3.9 in [18], we have

$$
\mathcal{W}\left(\theta_{v}: \mathcal{W}_{\max }(\mathbb{C}) \rightarrow \mathcal{W}(v)\right)_{c b} \leq \mathcal{O}\left(\theta_{v}\right)_{c b}=1
$$

and

$$
\mathcal{O}\left(\theta_{v}\right)_{c b} \leq \mathcal{W}\left(\theta_{v}: \omega(\mathbb{C}) \rightarrow \mathcal{W}(v)\right)_{c b}
$$

So

$$
\mathcal{W}\left(\theta_{v}: \omega(\mathbb{C}) \rightarrow \mathcal{W}(v)\right)_{c b}=\mathcal{O}\left(\theta_{v}\right)_{c b}=1 .
$$

Now we consider the condition for finite dimensional numerical radius operator spaces.

Proposition 2.2. Given abstract operator spaces $\left(V, \mathcal{O}_{n}\right)$ and $\left(W, \mathcal{O}_{n}\right)$ with either $V$ or $W n$-dimensional, $\left(V, \mathcal{W}_{n}\right)$ and $\left(W, \mathcal{W}_{n}\right)$ are numerical radius operator spaces, any linear mapping $\varphi: V \rightarrow W$ satisfies

$$
\mathcal{W}(\varphi: \mathcal{W}(V) \rightarrow \mathcal{W}(W))_{c b} \leq n \mathcal{W}(\varphi: \mathcal{W}(V) \rightarrow \mathcal{W}(W)) .
$$

Proof. Let us suppose that $W$ has dimension $n$. We may select an Auerbach basis for $W$, which by definition is a vector basis $w_{1}, w_{2}, \cdots, w_{n}$ with $\mathcal{W}\left(w_{j}\right)=1$, there exist $g_{j} \in \mathcal{W}(W)^{*}$ with $\mathcal{W}\left(g_{j}\right)=1$ and $g_{j}\left(w_{i}\right)=\delta_{i j}$. Since

$$
i d_{W}=\sum_{j=1}^{n} \theta_{w_{j}} \circ g_{j}
$$

We have

$$
\varphi=\sum_{j=1}^{n} \theta_{w_{j}} \circ g_{j} \circ \varphi,
$$

where $\theta_{w_{j}}(\alpha)=\alpha w_{j}$ are $\mathcal{W}$-complete isometries from $\mathbb{C}$ to $W$, and $g_{j} \circ \varphi$ are bounded linear functionals on $V$. It follows from Lemma 2.3 in [18] that

$$
\begin{aligned}
& \mathcal{W}(\varphi: \mathcal{W}(V) \rightarrow \mathcal{W}(W))_{c b} \\
\leq & \sum_{j=1}^{n} \mathcal{W}\left(\theta_{w_{j}}: \omega(\mathbb{C}) \rightarrow \mathcal{W}(W)\right)_{c b} \cdot \mathcal{W}\left(g_{j} \circ \varphi: \mathcal{W}(V) \rightarrow \omega(\mathbb{C})\right)_{c b} \\
= & \sum_{j=1}^{n} \mathcal{W}\left(g_{j} \circ \varphi: \mathcal{W}(V) \rightarrow \omega(\mathbb{C})\right) \\
\leq & n \mathcal{W}(\varphi: \mathcal{W}(V) \rightarrow \mathcal{W}(W)) .
\end{aligned}
$$

Similarly, if $V$ is $n$-dimensional, then we may replace $W$ by $\varphi(W)$, which has dimension less than or equal to $n$, and the result follows from the previous argument. 
Proposition 2.3. If $\left(V, \mathcal{O}_{n}\right)$ and $\left(W, \mathcal{O}_{n}\right)$ are n-dimensional operator spaces, $\left(V, \mathcal{W}_{n}\right),\left(W, \mathcal{W}_{n}\right)$ are numerical radius operator spaces, then there exists a linear isomorphism $\varphi: \mathcal{W}(V) \rightarrow \mathcal{W}(W)$ such that

$$
\mathcal{W}(\varphi: \mathcal{W}(V) \rightarrow \mathcal{W}(W))_{c b} \cdot \mathcal{W}\left(\varphi^{-1}: \mathcal{W}(W) \rightarrow \mathcal{W}(V)\right)_{c b} \leq n^{2}
$$

Proof. We choose Auervach bases $v_{i} \in V$ and $w_{i} \in W(i=1, \cdots, n)$, together with dual bases $f_{i} \in \mathcal{W}(V)^{*}$ and $g_{i} \in \mathcal{W}(W)^{*}$ with $\mathcal{W}\left(f_{i}\right)=\mathcal{W}\left(g_{i}\right)=1$. We have that

$$
\varphi: V \rightarrow W: v \mapsto \sum_{i=1}^{n} f_{i}(v) w_{i}
$$

and

$$
\psi: W \rightarrow V: w \mapsto \sum_{i=1}^{n} g_{i}(w) v_{i}
$$

are inverse linear mappings. Since

$$
\begin{aligned}
& \mathcal{W}(\varphi: \mathcal{W}(V) \rightarrow \mathcal{W}(W))_{c b} \\
& \leq \sum_{i=1}^{n} \mathcal{W}\left(f_{i}: \mathcal{W}(V) \rightarrow \omega(\mathbb{C})\right)_{c b} \cdot \mathcal{W}\left(\theta_{w_{i}}: \omega(\mathbb{C}) \rightarrow \mathcal{W}(W)\right)_{c b} \leq n
\end{aligned}
$$

and similarly

$$
\mathcal{W}(\psi: \mathcal{W}(W) \rightarrow \mathcal{W}(V))_{c b} \leq n,
$$

the result follows.

For any commutative $\mathrm{C}^{*}$-algebra, we can assume that $\mathcal{A}$ coincides with $C_{0}(\Omega)$. We may identify $M_{n}\left(C_{0}(\Omega)\right)$ with $C_{0}\left(\Omega, M_{n}\right)$. When given $f=\left[f_{i j}\right] \in M_{n}\left(C_{0}(\Omega)\right)$, we define

$$
\mathcal{W}(f)=\sup _{w \in \Omega}\left\{\omega\left(f_{i j}(w)\right)\right\},
$$

then $C_{0}(\Omega)$ can be seen as a numerical radius operator space. We call such $\mathcal{A}$ a commutative $\mathrm{C}^{*}$-algebra with a numerical radius norm.

Theorem 2.4. Let $V$ be a numerical radius operator space, and let $\mathcal{A}$ be a commutative $\mathrm{C}^{*}$-algebra with a numerical radius norm. Then any bounded linear mapping $\varphi: V \rightarrow \mathcal{A}$ satisfies $\mathcal{W}(\varphi)_{c b}=\mathcal{W}(\varphi)$.

Proof. We can assume that $\mathcal{A}$ coincides with $C_{0}(\Omega)$. Taking the supremum over all $w \in \Omega$ and $\alpha \in \mathbb{C}^{n}$ with $\|\alpha\|_{2}=1$, we have

$$
\begin{aligned}
& \mathcal{W}\left(\varphi_{n}(v)\right)=\mathcal{W}\left(\varphi\left(v_{i, j}\right)\right)=\sup _{w \in \Omega}\left\{\omega\left(\varphi\left(v_{i, j}\right)(w)\right)\right\} \\
& =\sup _{w, \alpha}\left\{\left\langle\varphi\left(v_{i, j}\right)(w) \alpha \mid \alpha\right\rangle\right\}=\sup _{w, \alpha}\left\{\left|\sum \bar{\alpha}_{i} \varphi\left(v_{i, j}\right)(w) \alpha_{j}\right|\right\}
\end{aligned}
$$

and thus letting $\alpha$ also stand for column matrices,

$$
\mathcal{W}\left(\varphi_{n}(v)\right)=\sup _{w, \alpha}\left\{\left|\varphi\left(\alpha^{*} v \alpha\right)(w)\right|\right\} \leq \mathcal{W}(\varphi) \sup _{\alpha}\left\{\mathcal{W}\left(\alpha^{*} v \alpha\right)\right\} \leq \mathcal{W}(\varphi) \mathcal{W}(v) .
$$

This shows that that $\mathcal{W}\left(\varphi_{n}\right) \leq \mathcal{W}(\varphi)$ for all $n \in \mathbb{N}$, and thus $\mathcal{W}(\varphi)_{c b}=\mathcal{W}(\varphi)$. 


\section{Dual Spaces of Numerical Radius Operator Spaces}

In this section, we introduce a lemma first.

Lemma 3.1. Suppose that $V$ is a numerical radius operator space. Given any element $v \in M_{n}(V)$, there exists a $\mathcal{W}$-complete contraction $\varphi: V \rightarrow M_{n}$ such that $\omega\left(\varphi_{n}(v)\right)=\mathcal{W}(v)$.

Proof. If we are given $v \in M_{n}(V)$, then we may use the Hahn-Banach theorem to find a linear functional $F \in\left[M_{n}(V)\right]^{*}$ with $\mathcal{W}^{*}(F)=1$ and $|F(v)|=\mathcal{W}(v)$. From Lemma 2.4 in [18], there is a corresponding $\mathcal{W}$-complete contraction $\varphi: V \rightarrow M_{n}$ for which

$$
\omega\left(\varphi_{n}(v)\right) \geq\left|\left\langle\varphi_{n}(v) \xi, \xi\right\rangle\right|=|F(v)|=\mathcal{W}(v) .
$$

The reverse inequality is trivial.

There is a natural numerical radius operator space structure on the mapping space $\mathcal{W} C B(V, W)$. In this paper, we consider the dual space

$$
V^{*}=B(V, \mathbb{C})=\mathcal{W} C B(V, \mathbb{C}) .
$$

Our task is to define $M_{n}\left(V^{*}\right)$ by introducing an appropriate norm on $M_{n}\left(V^{*}\right)$.

Each $f=\left[f_{i, j}\right] \in M_{n}\left(V^{*}\right)$ determines a linear mapping $f: V \rightarrow M_{n}$, where $f(v)=\left[f_{i, j}(v)\right]$. This gives us a linear isomorphism $M_{n}\left(V^{*}\right) \cong \mathcal{W} C B\left(V, M_{n}\right)$, which we use to determine the norm on $M_{n}\left(V^{*}\right)$. Thus, if we let $M_{n}\left(V^{*}\right)$ be the corresponding normed space, we have the isometric identification

$$
M_{n}\left(V^{*}\right)=\mathcal{W} C B\left(V, M_{n}\right) \text {. }
$$

For any $f \in M_{n}\left(V^{*}\right)$, we have from Lemma 2.3 in [18],

$$
\begin{aligned}
\mathcal{W}(f) & =\sup \left\{\omega\left(f_{n}(v)\right): v \in M_{n}(V), \mathcal{W}(v) \leq 1\right\} \\
& =\sup \left\{\omega(\langle\langle f, v\rangle\rangle): v \in M_{n}(V), \mathcal{W}(v) \leq 1\right\},
\end{aligned}
$$

where $\langle\langle\cdot, \cdot\rangle\rangle$ is the matrix pairing. Conversely, the norm on $M_{n}\left(V^{*}\right)$ determines that on $M_{n}(V)$. Since we have from Lemma 3.1 that for any $v \in M_{n}(V)$,

$$
\begin{aligned}
\mathcal{W}(v) & =\sup \left\{\omega\left(f_{n}(v)\right): f \in \mathcal{W} C B\left(V, M_{n}\right), \mathcal{W}(f)_{c b} \leq 1\right\} \\
& =\sup \left\{\omega(\langle\langle f, v\rangle\rangle): f \in \mathcal{W} C B\left(V, M_{n}\right), \mathcal{W}(f)_{c b} \leq 1\right\} .
\end{aligned}
$$

Proposition 3.2. The matrix norms on $V^{*}$ determine a numerical radius operator space.

Proof. Let us suppose that we are given $f \in M_{n}\left(V^{*}\right), \quad \alpha \in M_{n, m}$. Then

$$
\begin{aligned}
\mathcal{W}\left(\left(\alpha f \alpha^{*}\right)_{r}\right) & =\mathcal{W}\left(\left(\alpha \otimes I_{r}\right) f_{r}\left(\alpha \otimes I_{r}\right)^{*}\right) \\
& \leq\left\|\alpha \otimes I_{r}\right\|^{2} \mathcal{W}\left(f_{r}\right) \\
& \leq\|\alpha\|^{2} \mathcal{W}(f)_{c b},
\end{aligned}
$$

and hence $\mathcal{W}\left(\alpha f \alpha^{*}\right)_{c b} \leq\|\alpha\|^{2} \mathcal{W}(f)_{c b}$. We have WII.

On the other hand, given $f \in M_{n}\left(V^{*}\right), g \in M_{n}\left(V^{*}\right)$, and $v \in M_{r}(V)$ with $\mathcal{W}(v) \leq 1$, 


$$
\begin{aligned}
\omega\left((f \oplus g)_{r}(v)\right) & =\omega\left(\left[f\left(v_{i, j}\right) \oplus g\left(v_{i, j}\right)\right]\right) \\
& =\omega\left(f_{r}(v) \oplus g_{r}\left(v_{i, j}\right)\right) \\
& =\max \left\{\omega\left(f_{r}(v)\right), \omega\left(g_{r}(v)\right)\right\} \\
& \leq \max \left\{\mathcal{W}(f)_{c b}, \mathcal{W}(g)_{c b}\right\},
\end{aligned}
$$

and hence $\mathcal{W}(f \oplus g)_{c b} \leq \max \left\{\mathcal{W}(f)_{c b}, \mathcal{W}(g)_{c b}\right\}$. We have WI.

If $\varphi: V \rightarrow W$ is $\mathcal{W}$-completely bounded mapping, then we let $\varphi^{*}: W^{*} \rightarrow V^{*}$ be the dual Banach space mapping. For any $v \in M_{n}(V)$ and $g \in M_{m}\left(W^{*}\right)$, we have

$$
\left\langle\left\langle g, \varphi_{n}(v)\right\rangle\right\rangle=\left[g_{k, l}\left(\varphi\left(v_{i, j}\right)\right)\right]=\left[\varphi^{*}\left(g_{k, l}\right)\left(v_{i, j}\right)\right]=\left\langle\left\langle\left(\varphi^{*}\right)_{m}(g), v\right\rangle\right\rangle .
$$

Proposition 3.3. Given numerical radius operator spaces $V$ and $W$, and a $\mathcal{W}$ -completely bounded mapping $\varphi: V \rightarrow W$, we have $\mathcal{W}\left(\left(\varphi^{*}\right)_{n}\right)=\mathcal{W}\left(\varphi_{n}\right)$ for all $n \in \mathbb{N}$, and $\mathcal{W}\left(\varphi^{*}\right)_{c b}=\mathcal{W}(\varphi)_{c b}$.

Proof. The second relation is immediate from the first. The first follows from the calculation

$$
\mathcal{W}\left(\left(\varphi^{*}\right)_{n}\right)=\sup \left\{\omega\left\langle\left\langle\left(\varphi^{*}\right)_{n}(g), v\right\rangle\right\rangle\right\}=\sup \left\{\mathcal{W}\left(\left\langle\left\langle g, \varphi_{n}(v)\right\rangle\right\rangle\right)\right\}=\mathcal{W}\left(\varphi_{n}\right),
$$

where the supermum is taken over all $g \in M_{n}\left(W^{*}\right)$ and $v \in M_{n}(v)$ of norm less than 1.

We also note that given a $\mathcal{W}$-completely bounded mapping $\varphi \in \mathcal{W} C B(V, W)$, its second adjoint mapping $\varphi^{* *}: V^{* *} \rightarrow W^{* *}$ is in $\mathcal{W} C B\left(V^{* *}, W^{* *}\right)$ with $\mathcal{W}\left(\varphi^{* *}\right)_{c b}=\mathcal{W}(\varphi)_{c b}$, where $\varphi^{* * *}$ restricted to $V$ is equal to $\varphi$.

Given a numerical radius operator space $W$ which is the dual of a complete numerical radius operator space $V$, and a Hilbert space $\mathcal{H}$, we say that a mapping $\varphi: W \rightarrow(\mathcal{B}(\mathcal{H}), \omega)$ is a dual realization of $W$ on $\mathcal{H}$, if it is a weak ${ }^{\star}$ homeomorphic $\mathcal{W}$-completely isometric injection.

Theorem 3.4. If $V$ is a complete numerical radius operator space, then $V^{*}$ has a dual realization on a Hilbert space $\mathcal{H}$.

Proof. Let $s_{n}^{\sigma}=M_{n}(V)_{\mathcal{W}(\cdot) \leq 1}$. We have from Lemma 2.3 [18] that if $f \in M_{n}\left(V^{*}\right)=\mathcal{W} C B\left(V, M_{n}\right)$, then $\mathcal{W}(f)=\sup \left\{\omega(\langle\langle\varphi, f\rangle\rangle), \varphi \in s_{n}^{\sigma}\right\}$. We define $s^{\sigma}=\bigcup_{n \in \mathbb{N}} s_{n}^{\sigma}$ and we let $\mathcal{H}=\oplus_{\varphi \in s^{\sigma}} \mathbb{C}^{n(\varphi)}$, where $n=n(\varphi)$ is the integer with $\varphi \in s_{n}^{\sigma}$. The argument in the proof of Theorem 2.1 in [18] shows that the mapping

$$
\Phi: V^{*} \rightarrow \mathcal{B}(\mathcal{H}): f \rightarrow(\varphi(f))_{\varphi \in s^{\sigma}}
$$

is a $\mathcal{W}$-complete isometry. It is obvious that the mapping $\Phi$ is continuous in the weak ${ }^{*}$ topology. Since $V_{\mathcal{W}(\cdot) \leq 1}$ is weak ${ }^{*}$ compact, then its domain $\Phi\left(V_{\mathcal{W}(\cdot) \leq 1}^{*}\right)$ is also weak ${ }^{*}$ compact and is a closed subspace of $(\mathcal{B}(\mathcal{H}), \omega)$. Finally, $\Phi$ is 
one-to-one and weak $^{*}$ continuous on $V_{\mathcal{W}(\cdot) \leq 1}^{*}$, thus it is a weak ${ }^{*}$ homeomorphism. Since $V$ is complete, $\Phi$ maps $V^{*}$ weak $^{\star}$ homeomorphically onto its image.

Proposition 3.5. If $W$ is complete, then so is $\mathcal{W} C B(V, W)$.

Proof. Let us suppose that $W$ is complete. It suffices to show that $\mathcal{W} C B(V, W)$ is a closed subspace of $B(V, W)$. Given any Cauchy sequence $\varphi_{n} \in \mathcal{W} C B(V, W)$, it is clear that $\varphi_{n}$ is a Cauchy sequence in $B(V, W)$. From classical Banach space theory, $B(V, W)$ is complete, and thus there is a bounded linear mapping $\varphi: V \rightarrow W$ such that $\varphi_{n}$ converges to $\varphi$ in the norm topology, i.e., $\mathcal{W}\left(\varphi_{n}-\varphi\right) \rightarrow 0$. Since $\varphi_{n}$ is Cauchy in $\mathcal{W} C B(V, W)$, for any $\varepsilon>0$ there exist a sufficiently large integer $N(\varepsilon)>0$ such that whenever $n, m>N(\varepsilon)$, we have

$$
\mathcal{W}\left(\varphi_{n}-\varphi_{m}\right)_{c b}<\varepsilon
$$

Given any $v=\left[v_{i, j}\right] \in M_{p}(V)$ and $p \in N$, we have

$$
\mathcal{W}\left(\left(\varphi_{n}-\varphi_{m}\right)_{p}(v)\right) \leq \mathcal{W}\left(\varphi_{n}-\varphi_{m}\right)_{c b} \cdot \mathcal{W}(v)<\varepsilon \mathcal{W}(v)
$$

Since $\varphi_{m}\left(v_{i, j}\right)$ converges to $\varphi\left(v_{i, j}\right)$ in $W$, we have

$$
\mathcal{W}\left(\left(\varphi_{n}-\varphi\right)_{p}(v)\right) \leq \varepsilon \mathcal{W}(v),
$$

and thus $\mathcal{W}\left(\varphi_{n}-\varphi\right)_{c b} \leq \varepsilon$. It follows that $\varphi \in \mathcal{W} C B(V, W)$ and $\varphi_{n}$ converges to $\varphi$ in $\mathcal{W} C B(V, W)$.

\section{The Min and Max Numerical Radius Operator Spaces}

We let $\eta$ denote the category of normed spaces, in which the objects are the normed spaces and the morphisms are the bounded linear mappings. Similarly, we let $\mathfrak{D}$ be the category of numerical radius operator spaces with the morphisms being the $\mathcal{W}$-completely bounded mappings. We have a natural "forgetful" functor $N: \mathfrak{D} \rightarrow \eta$ which maps a numerical radius into its underlying normed space. We say that a functor $Q: \eta \rightarrow \mathfrak{D}$ is a strict quantization if for each normed space $E, N \circ Q(E)=E$, and for each bounded linear mapping of normed space $\varphi: E \rightarrow F$, the corresponding mapping $Q(\varphi): Q(E) \rightarrow Q(F)$ satisfies $\mathcal{W}(Q(\varphi))_{c b}=\mathcal{W}(\varphi)$.

For any Banach space $E$, we let $b_{r}(E)=B\left(E, M_{r}\right)_{\mathcal{W}(\cdot) \leq 1}$ and $b(E)=\bigcup_{r \in N} b_{r}(E)$. We define the matrix norms $\mathcal{W}(v)_{\text {Min }}$ and $\mathcal{W}(v)_{\text {Max }}$ for $v \in M_{n}(E)$ by

$$
\mathcal{W}(v)_{\text {Min }}=\sup \left\{\omega\left(f_{n}(v)\right): f \in b(E)\right\}
$$

and

$$
\mathcal{W}(v)_{\text {Max }}=\sup \left\{\omega\left(f_{n}(v)\right): f \in b_{1}(E)\right\}
$$

Proposition 4.1. $\left(V, \mathcal{W}_{\text {Min }}\right)$ and $\left(V, \mathcal{W}_{\text {Max }}\right)$ are both numerical radius oper- 
ator spaces.

Proof. To see that these are indeed numerical radius operator space matrix norms, it suffices to consider the linear injections

$$
E \rightarrow \ell_{\infty}\left(b_{1}(E)\right): V \rightarrow(f(v))_{f \in b_{1}(E)}
$$

and

$$
E \rightarrow \prod_{r \in \mathbb{N}} \ell_{\infty}\left(b_{r}(E), M_{r}\right): V \rightarrow\left((f(v))_{f \in b_{r}(E)}\right)_{r \in \mathbb{N}}
$$

respectively. We have the natural numerical radius operator space identifications $\ell_{\infty}\left(b_{1}(E)\right)=\prod_{b_{1}(E)} \mathbb{C}$ and $\prod_{r \in \mathbb{N}} \ell_{\infty}\left(b_{r}(E), M_{r}\right)=\prod_{r \in \mathbb{N}} \prod_{b_{r}(E)} M_{r}$.

Since the relative matrix norms on $E$ are given above, it is evident that these determine numerical radius operator spaces, which we denote by Min $E$ and $\operatorname{Max} E$, respectively. We refer to these numerical radius operator spaces as the minimal and the maximal quantization of $E$.

If $V$ is a numerical radius operator space and $v \in M_{n}(V)$, then it follows from Lemma 2.3 in [18] that

$$
\mathcal{W}(v)=\sup \left\{\omega\left(f_{n}(v)\right): f \in s_{n}(V)\right\} .
$$

Since $b_{1}(v) \subseteq s_{n}(v) \subseteq b(v)$, we conclude that $\mathcal{W}(v)_{\text {Min }} \leq \mathcal{W}(v) \leq \mathcal{W}(v)_{\text {Max }}$ for any $v \in M_{n}(V)$.

Proposition 4.2. For any numerical radius operator space $V$ and normed space $E$, and any linear mapping $\varphi: V \rightarrow E$, we have

$$
\mathcal{W} C B(V, M i n E)=\mathcal{W}(\varphi: V \rightarrow \operatorname{MinE})_{c b}=\mathcal{W}(\varphi: V \rightarrow E) .
$$

Proof. Let us suppose that $v \in M_{n}(V)$ and $\mathcal{W}(v) \leq 1$. Then

$$
\mathcal{W}\left(\varphi_{n}(v)\right)_{\text {Min }}=\sup \left\{\mathcal{W}\left(f_{n} \circ \varphi_{n}(v)\right): \mathcal{W}(f: E \rightarrow \mathbb{C}) \leq 1\right\} .
$$

But $\mathcal{W}(f) \leq 1$ implies that

$$
\mathcal{W}\left((f \circ \varphi)_{n}\right) \leq \mathcal{W}(f \circ \varphi)_{c b}=\mathcal{W}(f \circ \varphi) \leq \mathcal{W}(f) \cdot \mathcal{W}(\varphi) \leq \mathcal{W}(\varphi)
$$

and thus $\mathcal{W}\left(\varphi_{n}(v)\right)_{\text {Min }} \leq \mathcal{W}(\varphi)$ for all $n \in \mathbb{N}$. The inversion is clear.

If $\varphi: E \rightarrow F$ is a contraction, then since $\varphi: M i n E \rightarrow M i n F$ is a contraction,

$$
\operatorname{Min} \varphi=\varphi: \operatorname{MinE} \rightarrow \operatorname{MinF}
$$

is $\mathcal{W}$-completely contractive. We conclude that $M i n$ is a strict quantization functor. If $\varphi: E \rightarrow F$ is an isometric injection, then it follows that $\varphi$ is $\mathcal{W}$ -completely isometric since we may extend any $f \in b_{1}(E)$ to a functional $\tilde{f} \in b_{1}(F)$.

Proposition 4.3. For any normed space $E$ and numerical radius operator space $W$, we have

$$
\mathcal{W} C B(\operatorname{Max} E, W)=B(E, W) \text {, }
$$

i.e., for any linear mapping $\varphi: E \rightarrow W$,

$$
\mathcal{W}(\varphi: \operatorname{Max} E \rightarrow W)_{c b}=\mathcal{W}(\varphi: E \rightarrow W) .
$$


Proof. To prove this, it suffices to show that if $\mathcal{W}(\varphi) \leq 1$, then $\mathcal{W}(\varphi)_{c b} \leq 1$. For any $n \in \mathbb{N}$ and $v \in M_{n}(\operatorname{Max} E)$, we have

$$
\begin{aligned}
& \mathcal{W}\left(\varphi_{n}(v)\right) \\
& =\sup \left\{\mathcal{W}\left(f_{n} \circ \varphi_{n}(v)\right): \mathcal{W}\left(f: W \rightarrow M_{r}\right)_{c b} \leq 1, r \in \mathbb{N}\right\} \\
& \leq \sup \left\{\mathcal{W}\left((f \circ \varphi)_{n}(v)\right): \mathcal{W}\left(f: W \rightarrow M_{r}\right) \leq 1, r \in \mathbb{N}\right\} \\
& \leq \sup \left\{\mathcal{W}\left(g_{n}(v)\right): \mathcal{W}\left(g: E \rightarrow M_{r}\right) \leq 1, r \in \mathbb{N}\right\} \\
& =\mathcal{W}(v)_{\text {Max }} .
\end{aligned}
$$

From the above, we conclude that $\mathcal{W}(\varphi)_{c b} \leq 1$.

In particular, if we are given normed spaces $E$ and $F$ and a contraction $\varphi: E \rightarrow F$, since $\varphi: E \rightarrow \operatorname{Max} F$ is a contraction, we have

$$
\operatorname{Max} \varphi: \operatorname{Max} E \rightarrow \operatorname{MaxF}
$$

is a $\mathcal{W}$-complete contraction. Thus $\operatorname{Max}$ is a strict quantization.

If there is a contraction $\psi: F \rightarrow E$ such that $\psi \circ \varphi=i d_{E}$, then $\operatorname{Max} \varphi$ is $\mathcal{W}$ -completely isometric since $\operatorname{Max} \psi \circ \operatorname{Max} \varphi=i d_{M a x} E$. This is also the case for the canonical injection $E \hookrightarrow E^{* *}$, since any contraction $f: E \rightarrow M_{n}$ automatically extends to the contraction $f^{* *}: E^{* *} \rightarrow M_{n}$.

Proposition 4.4 If $D$ is a subset of $V_{\mathcal{W}(\cdot) \leq 1}^{*}$, and the absolutely convex hull $|c o|(D)$ is weak ${ }^{*}$ dense in $V_{\mathcal{W}(\cdot) \leq 1}^{*}$. Then for any $v \in M_{n}(V)$,

$$
\mathcal{W}(v)_{\text {Min }}=\sup \left\{\omega\left(f_{n}(v)\right): f \in D\right\} .
$$

Proof. Let us suppose that $\omega\left(f_{n}(v)\right) \leq 1$ for all $f \in D$. If $g \in \sum_{k} t_{k} f_{k}$ where $f_{k} \in D$ and $\sum_{k}\left|t_{k}\right| \leq 1$, then

$$
\omega\left(g_{n}(v)\right)=\omega\left(\sum_{k} t_{k}\left(f_{k}\right)_{n}(v)\right) \leq \sum_{k}\left|t_{k}\right| \leq 1
$$

For the absolutely convex hull $|c o|(D)$ is weak ${ }^{*}$ dense in $V_{\mathcal{W}(\cdot) \leq 1}^{*}$, given an arbitrary element $g \in V_{\mathcal{W}(\cdot) \leq 1}^{*}$, we may find a net $g_{\beta} \in|c o|(D)$ converging to $g$ in the weak ${ }^{*}$ topology. Then $g_{\beta}\left(v_{i, j}\right)$ converges to $g\left(v_{i, j}\right)$ in the numerical radius norm topology. It follows that $\omega\left(g_{n}(v)\right) \leq 1$, and thus $\mathcal{W}(v)_{\text {Min }} \leq 1$.

For any $v \in M_{n}(E)$, the linear mappings $\varphi_{v}: f \rightarrow\left[f\left(v_{i, j}\right)\right]$ are just the weak ${ }^{*}$ linear mappings from $E^{*}$ into $\left(M_{n}, \omega\right)$, and thus we have the isometric identification $M_{n}(\operatorname{Min} E)=B^{\sigma}\left(E^{*}, M_{n}\right)$.

Theorem 4.5. Suppose $E$ is a normed space, then $(\operatorname{MaxE})^{*}=M^{*} E^{*}$.

Proof. Given a normed space $E, n \in \mathbb{N}$ and a linear mapping $f: E \rightarrow M_{n}$, the second adjoint $f^{* *}$ provides an extension of $f$ to a weak ${ }^{\star}$ continuous mapping from $E^{* *}$ to $M_{n}$. This provides us with a natural identification $B\left(E, M_{n}\right)=B^{\sigma}\left(E^{* *}, M_{n}\right)$. Thus, we have the isometries

$$
M_{n}\left((\operatorname{Max} E)^{*}\right)=\mathcal{W} C B\left(\operatorname{Max} E, M_{n}\right)=B\left(E, M_{n}\right)=M_{n}\left(\operatorname{MinE}^{*}\right) .
$$

The result follows.

If $\Omega$ is a locally compact Hausdorff space and $Z=C_{0}(\Omega)$ is the corres- 
ponding commutative $\mathrm{C}^{*}$-algebra, then we have a natural mapping

$$
\delta: \Omega \rightarrow Z^{*}: \delta(x)(v)=v(x) .
$$

It is a simple consequence of the bipolar theorem that $|c o|(\delta(\Omega))$ is weak dense in $Z_{\mathcal{W}(\cdot) \leq 1}^{*}$. From our preceding observation, if $a=\left[a_{i j}\right]$ is an element of $M_{n}(Z)$, we have

$$
\mathcal{W}(a)_{\text {Min }}=\sup \left\{\omega\left(\left[f\left(a_{i j}\right)\right]\right): f \in \delta(\Omega)\right\}=\sup \left\{\omega\left(\left[a_{i j}(x)\right]\right): x \in \Omega\right\},
$$

i.e., $M_{n}(Z) \cong C_{0}\left(\Omega, M_{n}\right)$. We conclude that as a numerical radius operator space, $Z$ is just the minimal quantization of its underlying Banach space, i.e., $Z=\operatorname{MinZ}$.

Theorem 4.6. Suppose $E$ is a normed space, then $(M i n E)^{*}=M_{a x}{ }^{*}$.

Proof. Given a normed space $E$, and an isometric injection $E \hookrightarrow Z$, where $Z$ is a commutative $\mathrm{C}^{\star}$-algebra. We have a corresponding commutative diagram

$$
\begin{array}{cccc}
E & \rightarrow & Z \\
\downarrow & & \downarrow \\
E^{* *} & & \rightarrow & Z^{* *}
\end{array}
$$

where the first column is an isometry, the second column is a $\mathcal{W}$-complete isometry, and both rows are isometric. Since $Z^{* *}$ is a numerical radius operator space, it determines the minimal numerical radius operator space structure on $E^{* *}$, hence $(\operatorname{Min} E)^{* *}=\operatorname{Min} E^{* *}$. Thus, we have the $\mathcal{W}$-complete isometries

$$
\left(\operatorname{Max}^{*}\right)^{*}=\operatorname{MinE} E^{* *}=(\operatorname{MinE})^{* *},
$$

and since these identifications are compatible with the dualities, we have the $\mathcal{W}$ -complete isometry $\operatorname{MaxE}^{*}=(\operatorname{MinE})^{*}$.

\section{Conclusion}

In this paper, we study the bounded linear operators and the dual spaces of the numerical radius operator spaces. We found that many of the basic results about the numerical radius operator space can be inspired by the theory of operator space. In the future, we will study the tensor product theory and local property in the category of numerical radius operator spaces. We believe that the further developments of the numerical radius operator space theory could play an import role in the operator space theory as well as have its own intrinsic merit.

\section{Supported}

Project partially supported by the National Natural Science Foundation of China (No. 11701301).

\section{Conflicts of Interest}

The authors declare no conflicts of interest regarding the publication of this paper. 


\section{References}

[1] Arveson, W. (1969) Subalgebras of C-Algebras. Acta Mathematica, 123, 141-224. https://doi.org/10.1007/BF02392388

[2] Ruan, Z.-J. (1988) Subspaces of C-Algebras. Journal of Functional Analysis, 76, 217-230. https://doi.org/10.1016/0022-1236(88)90057-2

[3] Effros, E.G. and Ruan, Z.-J. (1988) Representations of Operator Bimodules and Their Applications. Journal of Operator Theory, 19, 137-157.

[4] Effros, E.G. and Ruan, Z.-J. (1988) On Matricially Normed Spaces. Pacific Journal of Mathematics, 132, 243-264. https://doi.org/10.2140/pjm.1988.132.243

[5] Blecher, D.P. and Paulsen, V.I. (1991) Tensor Products of Operator Spaces. Journal of Functional Analysis, 99, 262-292. https://doi.org/10.1016/0022-1236(91)90042-4

[6] Effros, E.G. and Ruan, Z.-J. (1991) A New Approach to Operator Spaces. Canadian Mathematical Bulletin, 34, 329-337. https://doi.org/10.4153/CMB-1991-053-x

[7] Effors, E.G., Ozawa, N. and Ruan, Z.-J. (2001) On Injectivity and Nuclearity for Operator Spaces. Duke Mathematical Journal, 110, 489-521. https://doi.org/10.1215/S0012-7094-01-11032-6

[8] Dong, Z. and Ruan, Z.-J. (2007) Weak Exactness for Dual Operator Spaces. Journal of Functional Analysis, 253, 373-397. https://doi.org/10.1016/j.jfa.2007.06.003

[9] Han, K.H. (2007) An Operator Space Approach to Condition C. Journal of Mathematical Analysis and Applications, 336, 569-576. https://doi.org/10.1016/j.jmaa.2007.02.074

[10] Wang, Y.Y. (2016) Condition $C_{\wedge}^{\prime}$ of Operator Spaces. Canadian Mathematical Bulletin, 60, 1-7. https://doi.org/10.4153/CMB-2016-064-3

[11] Amini, M., Medghalchi, A. and Nikpey, H. (2018) Globally Exact Operator Spaces. Glasnik Matematički, 53, 179-186. https://doi.org/10.3336/gm.53.1.12

[12] Effros, E.G. and Ruan, Z.-J. (2000) Operator Spaces, London Mathematical Society Monographs. New Series, Vol. 23, the Clarendon Press, Oxford University Press, New York.

[13] Pisier, G. (2003) Introduction to Operator Space Theory. London Mathematical Society Lecture Note Series, Vol. 294. Cambridge University Press, Cambridge. https://doi.org/10.1017/CBO9781107360235

[14] Kavruk, A., Paulsen, V., Todorov, I. and Tomforde, M. (2011) Tensor Produces of Operator Systems. Journal of Functional Analysis, 261, 267-299.

https://doi.org/10.1016/j.jfa.2011.03.014

[15] Kavruk, A., Paulsen, V., Todorov, I. and Tomforde, M. (2013) Quotients, Exactness, and Nuclearity in the Operator System Category. Advances in Mathematics, 235, 321-360. https://doi.org/10.1016/j.aim.2012.05.025

[16] Luthra, P. and Kumar, A. (2017) Embeddings and Envelopes of Exact Operator Systems. Bulletin of the Australian Mathematical Society, 96, 274-285. https://doi.org/10.1017/S0004972717000284

[17] Itoh, T. and Nagisa, M. (2006) The Numerical Radius Haagerup Norm and Hilbert Space Square Factorizations. Journal of the Mathematical Society of Japan, 58, 363-377. https://doi.org/10.2969/jmsj/1149166780

[18] Itoh, T. and Nagisa, M. (2006) Numerical Radius Norms on Operator Space. Journal of the London Mathematical Society, 74, 154-166.

https://doi.org/10.1112/S0024610706022794 